\title{
Design of energy saving film used in residential buildings
}

\author{
Rong Xiao-hong ${ }^{1, a} \quad$ Wang wen-liang', b \\ ${ }^{1}$ College of Industrial Technology, Xuzhou, 221140, China; \\ 2Department of Physics,Nanchang University, Nanchang 330031,china; \\ arxh823@126.com b w.l.wang@163.com
}

Keywords: Glass, building, energy saving film

Abstract: Glass materials are widely used in building, which can improve the lighting condition of the building, but often have some problems in actual use. To solve the problem of increasing heat and energy consumption, an energy saving film is gained in this paper based on optical thin film theory and optimization method. The design results show that it can maintain the good light of glass in visible band and the thermal suppression in infrared band.

\section{Introduction}

China is a rapidly developing country in the world facing rapid development of real estate industry. Compared with previous buildings, glass materials are widely used to an unprecedented degree[1-3]. The glass window can improve the lighting condition of the building, but often have the problems of stinging light, increasing heat and energy consumption. How to maintain the good light of glass, and to solve the problems that exist in the real estate to some extent has become an urgent problem should to be solved.

Optical thin film technology is an optical branch based on the development of film interference theory. This technique was first applied to the military field, but many civil problems were also solved well by optical thin film technology with the development of scientific research and the technological marketization[4-6]. At present, optical thin film applications are gradually penetrating into fields such as solar energy, microelectronics, aviation and nuclear technology, et al. In the field of real estate construction, optical film can still exert its characteristic spectral control ability[7,8].

In this paper, we design an energy saving film used in residential buildings. Than can operate at incident angle from $0^{\circ}$ to $60^{\circ}$ and over wavelength range from $380 \mathrm{~nm}$ to $2200 \mathrm{~nm}$. The designs are gained based on optical thin film theory and with the aid of optimization method. In section 2, we introduce the basic theory about optical thin film and energy saving film. The design and results are described in section 3, and in section 4 we give some conclusions.

\section{Theory}

Optical thin film filter is very natural and appropriate solutions to many optical engineering problems like reduction or enhancement of reflectance, selective spectral filtering of complex optical systems. Mathematically, an optical thin film is regard as a homogeneous plane parallel layer of essentially infinite extent, whose thickness is comparable to the wavelength of the incident radiation and which is mainly characterized by refractive index $\left(n_{i}\right)$, extinction coefficient $\left(k_{i}\right)$ and thickness $\left(d_{i}\right)$. Optical thin film system is a multiplayer thin film stack, which is a finite 
combination of such layers having different film constants. The configuration of optical thin film system can be simply shown in Fig.1.

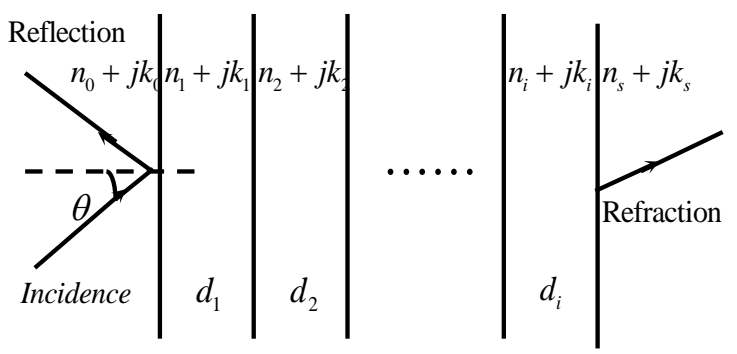

Fig.1. Configuration of optical thin film system

On the other hand, according to the study analysis, the energy of the solar spectral radiation is $55 \%$ in the infrared spectrum $(780-2200 \mathrm{~nm}), 44 \%$ in the visible band $(380-780 \mathrm{~nm})$, and only $3 \%$ in the ultraviolet band (100-380nm). If we can make a good control of the solar radiation spectrum by using optical thin film technology, we can achieve the spectral heat control of the sun. Based on this principle, the energy saving film can be designed to ensure the good light intensity in visible band and the thermal suppression in infrared band.

\section{Design and results}

\section{Optical coating Materials}

Considering the energy of the solar spectral radiation, the energy saving film we attempt to design in this paper should operate from $380 \mathrm{~nm}$ to $2200 \mathrm{~nm}$ spectral range. In this range, the coating materials have an appreciable dispersion that cannot be neglected and have to be handled properly through the whole design.
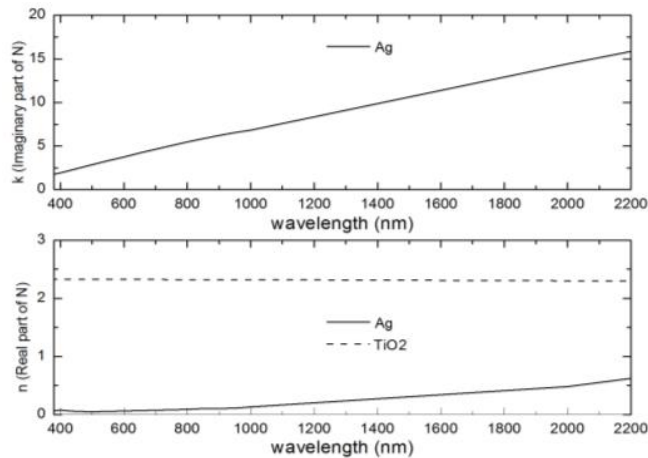

Fig. 2 optical materials used in design
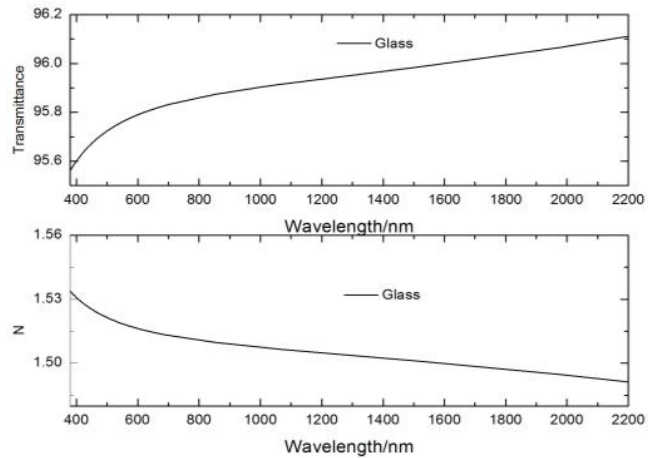

Fig.3 Optical constant and transmittance of glass

The optical coating materials we used in design are $\mathrm{TiO}_{2}$ and $\mathrm{Ag}$. Their refractive indices $(\mathrm{N}=\mathrm{n}+\mathrm{jk})$ used in the calculation are given in Fig. 2. As $\mathrm{TiO}_{2}$ coating material, the refractive index is relatively large and its change is small in the wide band range. But as Ag coating material, the refractive index is very small in the visible band and larger in the infrared band, especially its imaginary part. Then the optical constant and transmittance curve of glass is shown in Fig.3. We find that the dispersion of materials is very obvious, and the transmission rate in the infrared region is very large.

\section{design}

According to the distribution characteristics of solar spectral radiation energy, the good light intensity in visible band and the thermal suppression in infrared band should all must be considered 
in the design of energy saving film. As shown in section 3.1, $\mathrm{TiO}_{2}$ coating material has a high value refractive index in visible band, It is good for the high transmittance. And Ag coating material with a larger value about the imaginary part of refractive index in infrared band can inhibit thermal radiation better.

Then based on these analysis and optimization method, we design an energy saving film with the structure as $\mathrm{Glass}\left|\mathrm{TiO}_{2}(28.71 \mathrm{~nm}) \mathrm{Ag}(11.54 \mathrm{~nm}) \mathrm{TiO}_{2}(28.71 \mathrm{~nm})\right|^{\wedge}$ Air. The corresponding transmittance curve of our design result with $\mathrm{k}=1,3,5$ are shown in Fig. 4. We find that the transmittance in visible band is over $85 \%$ and the transmittance in infrared band is low. Moreover, the more the film layers, the lower the transmittance of the infrared band, while maintaining the high transmission rate of the visible band. It is obvious that the more layers, the stronger the inhibition of the infrared radiation, but the costs and technology have also increased. Maybe, $\mathrm{k}=3$ is best suitable.

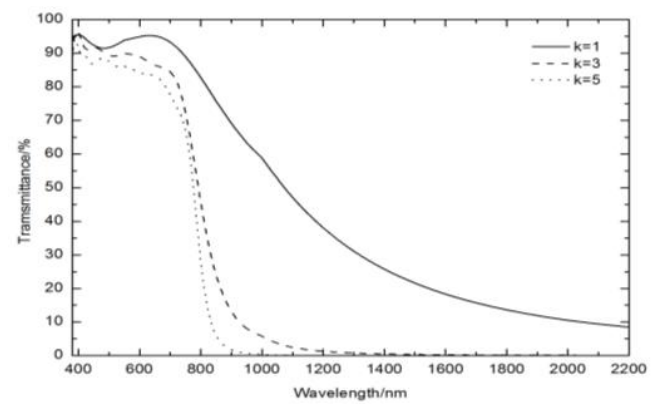

Fig.4 Transmittance curve of the design

\section{analysis}

In practice, the azimuth of the sun varies throughout the day. Therefore, we must consider the influence of the change of incidence angle on the performance of energy saving film. As shown in Fig.5, we can find that the transmittance of the visible band is over $80 \%$ When the incidence Angle is less than 60 degrees. But, when the incidence angle is increased to 80 degrees, the transmittance of the visible band is reduced to 50\%. In practical application, the angle of sunlight is below 60 degree during the main period of high temperature. So our design can meet the actual environmental requirements.

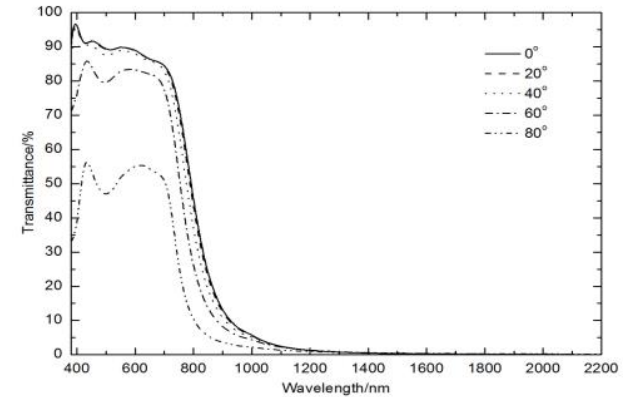

Fig.5 Transmittance curve of the design used in different incident angle

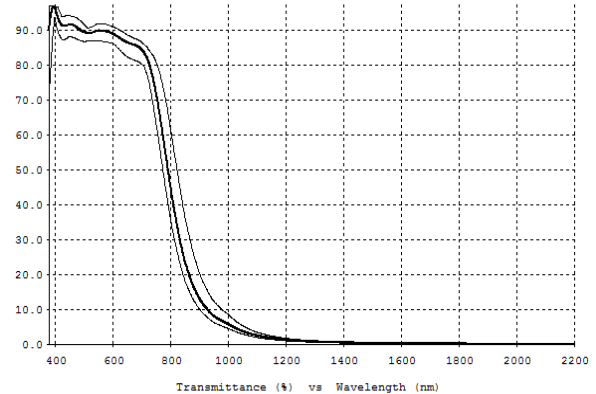

Fig.6 Layer thickness tolerance of the design

In addition, in actual manufacturing, the film layer thickness error is also inevitable. As shown in fig.6, when the film layer thickness has a random $\% 5$ relative error, the spectral curve changes only less than $3 \mathrm{~nm}$. So we can say, the design has a reasonable tolerance range.

\section{Conclusion}

How to maintain the good light of glass, and to solve the problems that exist in the real estate to some extent has become an urgent problem should to be solved. Considering $\mathrm{TiO}_{2}$ coating material 
has a high value refractive index in visible band, and Ag coating material has a larger value about the imaginary part of refractive index in infrared band, we design an energy saving film based on optical thin film theory and optimization method. Thou sun azimuth change continuously in a cycle of rising to setting, our design result can maintain the good light of glass in visible band and the thermal suppression in infrared band below incident angle 60 degree, which denote our design can meet the needs of actual environment. On the other hand, the spectral curve changes only less than $3 \mathrm{~nm}$ when the film layer thickness has a random $\% 5$ relative error. So we can say, the design has a reasonable tolerance range, and can be easy manufactured by using modern thin film technology.

\section{Acknowledgements}

This work is supported by the Research Foundation of Philosophy and Social Sciences in Universities of Jiangsu Province(2015SJB434), China.

\section{Reference}

[1] Zhitong Yao, Tung-Chai Ling, P.K. Sarker, Weiping Su, Jie Liu, Weihong Wu, Junhong Tang. Recycling difficult-to-treat e-waste cathode-ray-tube glass as construction and building materials: A critical review[J]. Renewable and Sustainable Energy Reviews, 2018, 81(1): 595-604.

[2] Bertha Alejandra Olmos Navarrete, Heriberto Puga Juárez, et. al. Failure behavior of annealed glass for building windows[J]. Engineering Structures, 2017,141(15): 417-426.

[3] G. Kiran Kumar, T.P. Ashok Babu. Study of Various Glass Materials to Provide Adequate Day Lighting in Office Buildings of Warm and Humid Climatic Zone in India[J]. Energy Procedia, 2017, 109: 181-189.

[4] Jennifer D. T. Kruschwitz* and Roy S. Berns. Nonpolarizing color mirrors on a high-reflecting metal base[J]. Applied optics, 2014, 53(16): 3448-3453.

[5] Weidong Zhou, Meng Tao, Li Chen, et al. Microstructured surface design for omnidirectional antireflection coatings on solar cells[J]. Journal of Applied Physics, 2007, 102,103105-1.

[6] Mao-Jung Huang, Chii-Rong Yang, Yuang-Cherng Chiou, et al. Fabrication of nanoporous antireflection surfaces on silicon[J]. Solar Energy Materials \& Solar cell, 2008,92: 1352-1357.

[7] Lazaros Elias Mavromatidis. Study of coupled transient radiation-natural convection heat transfer across rectangular cavities in the vicinity of low emissivity thin films for innovative building envelope applications[J]. Energy and Buildings, 2016, 120(15): 114-134.

[8] Chunying Li, Junyi Tan, Tin-Tai Chow, Zhongzhu Qiu. Experimental and theoretical study on the effect of window films on building energy consumption[J]. Energy and Buildings, 2015, 102(1): 129-138. 